\title{
Lattice of quasiorders of monounary algebras
}

\author{
D. Jakubiková-Studenovská
}




\title{
LATTICE OF QUASIORDERS OF MONOUNARY ALGEBRAS
}

\author{
D. JAKUBIKOVÁ-STUDENOVSKÁ
}

Received 24 November, 2008

\begin{abstract}
For an algebra $\mathcal{A}$, the lattice Quord $\mathcal{A}$ of all quasiorders of $\mathcal{A}$, i. e., of all reflexive and transitive relations compatible with all fundamental operations of $\mathcal{A}$, is dealt with. In the present paper we prove that if $\mathcal{A}$ is a monounary algebra, then Quord $\mathcal{A}$ is distributive if and only if it is modular and we find necessary and sufficient conditions for $\mathcal{A}$ under which Quord $\mathcal{A}$ is distributive.
\end{abstract}

2000 Mathematics Subject Classification: 08A60, 08A02, 08B99

Keywords: monounary algebra, quasiorder, lattice, modular, distributive

\section{INTRODUCTION}

A reflexive and transitive relation on an algebra $\mathcal{A}$, which is compatible with all fundamental operations of $\mathcal{A}$, is said to be a quasiorder of $\mathcal{A}$. Quasiorders of an algebra can be considered as a common generalization of its congruences and compatible partial orders.

The lattices of all quasiorders on a set have several special properties (e. g., they are atomistic, dually atomistic and complemented [4]). By [2,7], every algebraic lattice is isomorphic to the quasiorder lattice of a suitable algebra. Only few properties of the lattice of quasiorders are known in the case of concrete classes of algebras. For example, for the majority algebras it is known that their quasiorder lattice is always distributive $[3,8]$ ). In [9] the question how endomorphisms of quasiorders behave, in particular, under which conditions End $q \subseteq \operatorname{End} q^{\prime}$ for quasiorders $q, q^{\prime}$ on a set $A$ (End $q$ is the set of all mappings preserving $q$ ). They describe the quasiorder lattice of the algebra $(A, \operatorname{End} q)$.

We will deal with the lattice Quord $(A, f)$ of all quasiorders of $(A, f)$, where $(A, f)$ is a monounary algebra.

Let $(A, f)$ be a monounary algebra. Clearly, if $|A|=1$, then Quord $(A, f)$ is a 1element lattice and if $(A, f)$ is a 2-element cycle, then $\operatorname{Quord}(A, f)$ is a 2-element lattice. Also, we show that if $|A|=2$ and $(A, f)$ is not a cycle, then Quord $(A, f) \cong$ $M_{2}$.

Supported by Grant VEGA 1/3003/06. 
The aim of this paper is to find necessary and sufficient conditions under which the lattice Quord $(A, f)$ is modular or distributive, respectively.

\section{Preliminaries}

First we recall some basic notions. By a monounary algebra we understand a pair ( $A, f)$ where $A$ is a nonempty set and $f: A \rightarrow A$ is a mapping.

A monounary algebra $(A, f)$ is called connected if, for arbitrary $x, y \in A$, there are non-negative integers $n, m$ such that $f^{n}(x)=f^{m}(y)$. A maximal connected subalgebra of a monounary algebra is called a connected component.

An element $x \in A$ is referred to as cyclic, if there exists a positive integer $n$ such that $f^{n}(x)=x$. In this case the set $\left\{x, f^{1}(x), f^{2}(x), \ldots, f^{n-1}(x)\right\}$ is said to be a cycle.

A quasiorder of an algebra $\mathcal{A}=(A, F)$ is a reflexive and transitive binary relation on $A$, which is compatible with all operations $f \in F$. A quasiorder is a congruence of $\mathcal{A}$, if it is symmetric. We will denote by Quord $\mathcal{A}$ and $\operatorname{Con} \mathcal{A}$ the lattice of all quasiorders and of all congruences of $\mathcal{A}$, respectively, ordered by inclusion.

For an algebra $\mathcal{A}=(A, F), a, b \in A$, let $\alpha(a, b)$ and $\theta(a, b)$ be the smallest quasiorder and the smallest congruence, respectively, such that $(a, b) \in \alpha(a, b),(a, b) \in$ $\theta(a, b)$.

Trivial relations $I=\{(a, a): a \in A\}$ and $A^{2}$ are congruences. For a monounary algebra $(A, f)$, so called "natural ordering" $v$ defined for $x, y \in A$ by the formula $(x, y) \in v$ if $y=f^{n}(x)$ for some non-negative integer was studied, e. g., in [6]. It is easy to see that $v \in$ Quord $(A, f)$. Thus, e. g., if some connected component of $(A, f)$ has at least 3 elements, then $v$ is a nontrivial quasiorder.

If $\alpha \in$ Quord $(A, f), x \in A$, then we denote

$$
[x]_{\alpha}=\{y \in A:(x, y) \in \alpha,(y, x) \in \alpha\} .
$$

The symbols $\mathbb{N}$ and $\mathbb{Z}$ are used for the sets of all positive integers and of all integers. Next, if $n \in \mathbb{N}$, then $\mathbb{Z}_{n}$ is the system of all integers modulo $n$.

The pentagon $N_{5}$ and the diamond $M_{3}$ are used as in [5]; also, for $n \in \mathbb{N}, M_{n}$ is a lattice with $n+2$ elements $0,1, a_{1}, a_{2}, \ldots, a_{n}$ such that $0<a_{i}<1, a_{i} \nless a_{j}$ for any $i, j \in\{1, \ldots, n\}, i \neq j$.

Lemma 2.1. Let us assume that $(A, f)$ is a monounary algebra with $A=\{0,1\}$, $f(0)=f(1)=0$. Put $\alpha=I \cup\{(0,1)\}$ and $\beta=I \cup\{(1,0)\}$. Then Quord $(A, f)=$ $\left\{I, A^{2}, \alpha, \beta\right\} \cong M_{2}$.

Proof. If $(0,1) \in \gamma$ for some $\gamma \in$ Quord $(A, f)$, then $(f(0), f(1)) \in \gamma$ must be valid, i. e., $(0,1) \in \gamma$ implies $(0,0) \in \gamma$, which is trivially satisfied. Hence the smallest element $\gamma$ belonging to Quord $(A, f)$ and such that $(0,1) \in \gamma$ is equal to $\alpha$. Similarly, $\beta$ is the smallest quasiorder $\delta$ with the property that $(1,0) \in \delta$. The remaining members of Quord $(A, f)$ are $I$ and $A^{2}$. Hence Quord $(A, f) \cong M_{2}$. 
Lemma 2.2. If $(A, f)$ is a monounary algebra containing at least three 1-element cycles, then Quord $(A, f)$ contains a pentagon.

Proof. Let $a, b, c \in A$ be distinct elements of $A$ which form one-element cycles. Put

$$
\alpha_{1}=I \cup\{(b, a)\}, \quad \alpha_{2}=I \cup\{(b, a),(c, a)\},
$$

and

$$
\beta_{1}=I \cup\{(c, b)\}, \quad \beta_{2}=I \cup\{(c, b),(b, a),(c, a)\} .
$$

Then $\alpha_{1}, \alpha_{2}, \beta_{1}, \beta_{2} \in \operatorname{Quord}(A, f)$. Next, $\alpha_{1}<\alpha_{2}<\beta_{2}, \beta_{1}<\beta_{2}$ and $\alpha_{1} \wedge \beta_{1}=$ $\alpha_{2} \wedge \beta_{1}=I$. Next, $\alpha_{1} \leq \beta_{2}, \beta_{1} \leq \beta_{2}$, thus $\alpha_{1} \vee \beta_{1} \leq \beta_{2}$. If $(x, y) \in \beta_{2}-\left(\alpha_{1} \cup \alpha_{2}\right)$, then $(x, y)=(c, a)$. Since $(c, b) \in \beta_{1},(b, a) \in \alpha_{1}$, we get by transitivity that $(c, a) \in$ $\beta_{1} \vee \alpha_{1}$. Hence $\alpha_{1} \vee \beta_{1}=\beta_{2}$. Therefore, $\alpha_{2} \vee \beta_{1}=\beta_{2}$. Thus we have a pentagon consisting of the quasiorders $\left\{I, \alpha_{1}, \alpha_{2}, \beta_{1}, \beta_{2}\right\}$.

Corollary 2.1. Let $(A, f)$ be a monounary algebra, $|A| \geq 3, f(x)=x$ for each $x \in A$. The lattice Quord $(A, f)$ contains a pentagon.

Let us remark that the statement of 2.1 can be proved in a shorter way: In this case the lattice Quord $(A, f)$ coincides with the lattice of all quasiorders of a set $A$. It is known that if $|A| \geq 3$, then this lattice is not modular.

\section{CONNECTED MONOUNARY ALGEBRA}

From the paper of Berman [1] concerning congruences it follows that if $n \in \mathbb{N}$, then $\theta$ is a congruence relation of an $n$-element cycle $(C, f)$ if and only if there is $d \in \mathbb{N}$ such that $d$ divides $n$ and for each $x \in C$,

$$
[x]_{\theta}=\left\{x, f^{d}(x), \ldots, f^{\left(\frac{n}{d}-1\right) d}(x)\right\} .
$$

The congruence with this property will be denoted $\theta_{d}$. It is easy to verify that for each $x \in C, \theta_{d}$ is the smallest congruence containing the pair $\left(x, f^{d}(x)\right)$.

Lemma 3.1. Let $(A, f)$ be an n-element cycle, $n \in \mathbb{N}$. Then Quord $(A, f)=$ $\operatorname{Con}(A, f)=\left\{\theta_{d}: d / n\right\}$.

Proof. Without loss of generality we can assume that $A=\mathbb{Z}_{n}$ and that $f(x) \equiv$ $x+1(\bmod n)$ for $x \in \mathbb{Z}_{n}$. Since each congruence is a quasiorder of $(A, f)$, we have to prove that Quord $(A, f) \subseteq\left\{\theta_{d}: d / n\right\}$.

Let $\alpha \in \operatorname{Quord}(A, f)$. If $\alpha=I$, then $\alpha=\theta_{n}$. Suppose that $\alpha \neq I$, i. e., $\left(i_{1}, i_{2}\right) \in \alpha$ for some $i_{1}, i_{2} \in \mathbb{Z}_{n}, i_{1} \neq i_{2}$. This implies that $\left(f^{n-i_{1}}\left(i_{1}\right), f^{n-i_{1}}\left(i_{2}\right)\right) \in \alpha$. We have

$$
f^{n-i_{1}}\left(i_{1}\right)=f^{n-i_{1}}\left(f^{i_{1}}(0)\right)=f^{n}(0)=0,
$$

and thus there exists the smallest $d$ with $(0, d) \in \alpha$. Denote by $e$ the greatest common divisor of $d$ and $n$. Then the numbers $n_{1}=\frac{n}{e}$ and $d_{1}=\frac{d}{e}$ are mutually prime. If $j d \equiv j^{\prime} d \bmod n$ for some $0 \leq j \leq j^{\prime}<n_{1}$, then $n / j d-j^{\prime} d, n_{1} /\left(j-j^{\prime}\right) d_{1}$, thus 
$n_{1} / j-j^{\prime}$ which yields that $j=j^{\prime}$. Therefore, the elements $0, d, 2 d, \ldots,\left(n_{1}-1\right) d$ are distinct. Further,

$$
\begin{aligned}
(0, d) \in \alpha \Rightarrow(d, 2 d)= & \left(f^{d}(0), f^{d}(d)\right) \in \alpha \\
& \Rightarrow(2 d, 3 d) \in \alpha \Rightarrow \cdots \Rightarrow\left(\left(n_{1}-1\right) d, n_{1} d\right) \in \alpha .
\end{aligned}
$$

Since $n_{1} d=\frac{n}{e} d=n d_{1} \equiv 0 \bmod n$, we have $\left(\left(n_{1}-1\right) d, 0\right) \in \alpha$, therefore we obtain

$$
[0]_{\alpha} \supseteq\left\{0, d, 2 d, \ldots,\left(n_{1}-1\right) d\right\} .
$$

Assume that $\left(0, f^{m}(0)\right) \in \alpha$ for some $m \in \mathbb{N}$. There are $q \in \mathbb{N}, z \in \mathbb{N} \cup\{0\}$ such that $m=q d+z, 0 \leq z<d$. We have $(q d, 0) \in \alpha$ by (3.1), thus $\left(f^{z}(q d), f^{z}(0)\right) \in \alpha$, $(q d+z, z) \in \alpha,(m, z) \in \alpha$. Hence

$$
(0, m) \in \alpha,(m, z) \in \alpha \Rightarrow(0, z) \in \alpha,
$$

which, together with the assumption that $d$ was the smallest positive integer with $(0, d) \in \alpha$, implies that $z=0$. Then by (3.2), $(m, 0) \in \alpha, m \in[0]_{\alpha}$. In view of (3.1) then

$$
[0]_{\alpha}=\left\{0, d, 2 d, \ldots,\left(n_{1}-1\right) d\right\}=\left\{0, d, 2 d, \ldots,\left(\frac{n}{d}-1\right) d\right\} .
$$

This implies

$$
\begin{aligned}
{[1]_{\alpha} } & =\left\{1,1+d, 1+2 d, \ldots, 1+\left(\frac{n}{d}-1\right) d\right\}, \\
& \vdots \\
{[d-1]_{\alpha} } & =\left\{d-1, d-1+d, d-1+2 d, \ldots, d-1+\left(\frac{n}{d}-1\right) d\right\} .
\end{aligned}
$$

Therefore, $d / n$ and $\alpha=\theta_{d}$.

Corollary 3.1. Let $(A, f)$ be an n-element cycle for some $n \in N$. Then the lattice Quord $(A, f)$ is distributive.

Proof. By Lemma 3.1, the lattice Quord $(A, f)$ is isomorphic to the lattice $D_{n}$ of all divisors of $n$, since $\theta_{d_{1}} \subseteq \theta_{d_{2}}$ if and only if $d_{2} / d_{1}$.

Lemma 3.2. Let $(A, f)$ be a connected monounary algebra containing an $n$ element cycle $C,|A|=n+1 \geq 3$. Then

$$
\operatorname{Quord}(A, f) \cong \operatorname{Quord}(C, f) \times M_{2}=\operatorname{Con}(C, f) \times M_{2}
$$

and Quord $(A, f)$ is distributive.

Proof. We can suppose that $A=\mathbb{Z}_{n} \cup\{a\}, f(i) \equiv i+1 \bmod n$ for each $i \in \mathbb{Z}_{n}$, $f(a)=0$. By Lemma 3.1, Quord $\left(\mathbb{Z}_{n}, f\right)=\left\{\theta_{d}: d / n\right\}$. Let $\alpha \in \operatorname{Quord}(A, f)$. Then $\alpha^{\prime}=\alpha \cap \mathbb{Z}_{n}^{2}$ is a quasiorder of $\left(\mathbb{Z}_{n}, f\right)$ and there is $d$ dividing $n$ with $\alpha^{\prime}=\theta_{d}$. One of the following conditions is satisfied:

(1) $\alpha=\alpha^{\prime} \cup\{(a, a)\}$, 
(2) there is $y \in \mathbb{Z}_{n}$ with $(a, y) \in \alpha$,

(3) there is $z \in \mathbb{Z}_{n}$ with $(z, a) \in \alpha$.

Let (3.2) hold. Then $(f(a), f(y)) \in \alpha$, thus $(0, y+1) \in \alpha$. Hence $(0, y+1) \in \theta_{d}$, $d / y+1$ and then

$$
[y+1]_{\theta_{d}}=\left\{0, d, 2 d, \ldots,\left(\frac{n}{d}-1\right) d\right\}
$$

This implies

$$
[y]_{\theta_{d}}=\left\{d-1, d-1+d, \ldots, d-1+\left(\frac{n}{d}-1\right) d\right\} .
$$

Then $\left\{(a, t): t \in[y]_{\theta_{d}}\right\} \subseteq \alpha$. If there is $y^{\prime} \neq y$ with $y^{\prime} \in \mathbb{Z}_{n},\left(a, y^{\prime}\right) \in \alpha$, then $\left(y, y^{\prime}\right) \in \alpha^{\prime}$ and $[y]_{\theta_{d}}=\left[y^{\prime}\right]_{\theta_{d}}$. Thus $(a, t) \in \alpha$ if and only if $t \in\{a, d-1, d-1+$ $\left.d, \ldots, d-1+\left(\frac{n}{d}-1\right) d\right\}$. Analogously, if (3.3) is valid, then $(t, a) \in \alpha$ if and only if $t \in\left\{a, d-1, d-1+d, \ldots, d-1+\left(\frac{n}{d}-1\right) d\right\}$. Therefore, if (3.1) fails to holds, then exactly one of the following conditions is satisfied:

$$
\begin{aligned}
& \alpha=\alpha^{\prime} \cup\left\{(a, t): t \in\left\{a, d-1, d-1+d, \ldots, d-1+\left(\frac{n}{d}-1\right) d\right\}\right\}, \\
& \alpha=\alpha^{\prime} \cup\left\{(t, a): t \in\left\{a, d-1, d-1+d, \ldots, d-1+\left(\frac{n}{d}-1\right) d\right\}\right\}, \\
& \alpha=\alpha^{\prime} \cup\left\{(t, a),(a, t): t \in\left\{a, d-1, d-1+d, \ldots, d-1+\left(\frac{n}{d}-1\right) d\right\}\right\} .
\end{aligned}
$$

Let us define a mapping $\varphi$ : Quord $(A, f) \rightarrow \operatorname{Quord}\left(\mathbb{Z}_{n}, f\right) \times M_{2}$; the elements of $M_{2}$ are denoted by $0,1,2,3$ (0-the least, 1 -the greatest element of $\left.M_{2}\right)$. If $\alpha$ satisfies (3.1), then we put $\varphi(\alpha)=\left(\alpha^{\prime}, 0\right)$. If $\alpha$ satisfies (3.4), ((3.5), (3.6), respectively), then we put $\varphi(\alpha)=\left(\alpha^{\prime}, 2\right)\left(\varphi(\alpha)=\left(\alpha^{\prime}, 3\right), \varphi(\alpha)=\left(\alpha^{\prime}, 1\right)\right.$, respectively). It is a routine calculation to verify that $\varphi$ is a lattice isomorphism, which yields the required assertion.

Lemma 3.3. If $(A, f)$ is a connected monounary algebra fulfilling no of the assumptions of 3.1 and 3.2, $|A|>2$, then Quord $(A, f)$ contains a pentagon, thus it fails to be modular.

Proof. By the assumption, there exist distinct elements $0,1,2 \in A$ such that if we denote $C=\left\{f^{k}(0): k \in \mathbb{N} \cup\{0\}\right\}$, then $1,2 \notin C, f(1)=0$ and $f(2) \in C \cup\{1\}$.

Consider the following relations on the set $A$ :

$$
\begin{aligned}
& \alpha_{0}=I \cup C^{2} \cup\{1\} \times C, \\
& \alpha_{1}=I \cup C^{2} \cup\{1,2\} \times C, \\
& \alpha_{2}=I \cup C^{2} \cup\{1,2\} \times C \cup\{(2,1)\}
\end{aligned}
$$


and

$$
\begin{aligned}
& \beta_{1}=I \cup(C \cup\{1\})^{2}, \\
& \beta_{2}=I \cup(C \cup\{1\})^{2} \cup\{2\} \times(C \cup\{1\}) .
\end{aligned}
$$

The above assumptions imply that these relations are quasiorders of $(A, f)$. Further,

(1) $\alpha_{0}<\alpha_{1}<\alpha_{2}<\beta_{2}$,

(2) $\alpha_{0}<\beta_{1}<\beta_{2}$.

Let $(x, y) \in \alpha_{2} \wedge \beta_{1}, x \neq y$. Then either $(x, y) \in C \times C$ or $x=1, y \in C$. Thus

(3) $\alpha_{2} \wedge \beta_{1}=\alpha_{0}$.

Since $\alpha_{1}<\alpha_{2}$, then (3) yields

(4) $\alpha_{1} \wedge \beta_{1}=\alpha_{0}$.

Let us count $\alpha_{1} \vee \beta_{1}$. We have $\alpha_{1}<\beta_{2}, \beta_{1}<\beta_{2}$, thus $\alpha_{1} \vee \beta_{1} \leq \beta_{2}$. Let $(x, y) \in$ $\beta_{2},(x, y) \notin \alpha_{1},(x, y) \notin \beta_{1}$. Then $x=2, y=1$. We have $(2,0) \in \alpha_{1},(0,1) \in \beta_{1}$, which implies that $(2,1) \in \alpha_{1} \vee \beta_{1}$, i. e., $(x, y) \in \alpha_{1} \vee \beta_{1}$. Therefore,

(5) $\alpha_{1} \vee \beta_{1}=\beta_{2}$.

Next, $\alpha_{2}>\alpha_{1}$ yields in view of (5) that

(6) $\alpha_{2} \vee \beta_{1}=\beta_{2}$.

We have shown that Quord $(A, f)$ contains a pentagon, and, therefore, it is not modular.

\section{General CASE}

Assume that $|A|>2$ and that $(A, f)$ is non-connected. Then either $f(x)=x$ for each $x \in A$ or there is a connected component $B$ of $(A, f)$ such that $|B| \geq 2$. In the first case it was shown in Lemma 2.2 that Quord $(A, f)$ contains a pentagon.

In Lemmas 4.1 and 4.2 , and Corollary 4.1 , let $B, C$ be distinct connected components of $(A, f)$ such that $|B| \geq 2$. Then $B \cap C=\varnothing$. Let us put

$$
\alpha_{1}=I \cup B \times C, \quad \beta_{1}=I \cup C \times B
$$

and

$$
\alpha_{2}=I \cup B^{2} \cup B \times C, \quad \beta_{2}=I \cup(B \cup C)^{2}
$$

Since $|B| \geq 2$, these relations are mutually distinct.

Lemma 4.1. The relations $\alpha_{1}, \alpha_{2}, \beta_{1}, \beta_{2}$ belong to $\operatorname{Quord}(A, f)$.

Proof. It is easy to see that each of the relations is reflexive and transitive. Let $x, y \in A, x \neq y$. If $(x, y) \in \alpha_{1}$, then $x \in B, y \in C$, thus $f(x) \in B, f(y) \in C$ and $(f(x), f(y)) \in \alpha_{1}$. Hence $\alpha_{1} \in \operatorname{Quord}(A, f)$. The remaining assertions can be proved analogously.

Lemma 4.2. The following relations assertions hold: 
(i) $\alpha_{1}<\alpha_{2}<\beta_{2}, \beta_{1}<\beta_{2}$,

(ii) $\alpha_{2} \wedge \beta_{1}=\alpha_{1} \wedge \beta_{1}=I$,

(iii) $\alpha_{1} \vee \beta_{1}=\alpha_{2} \vee \beta_{1}=\beta_{2}$.

Proof. The validity of (i) and (ii) is obvious. We have $\alpha_{1}<\beta_{2}, \beta_{1}<\beta_{2}$, thus $\alpha_{1} \vee \beta_{1} \leq \beta_{2}$. Let $(u, v) \in \beta_{2}$. If $u=v$, then $(u, v) \in \alpha_{1} \vee \beta_{1}$. If $(u, v) \in B \times C$ or $(u, v) \in C \times B$, then $(u, v) \in \alpha_{1}$ or $(u, v) \in \beta_{1}$, thus $(u, v) \in \alpha_{1} \vee \beta_{1}$. If $(u, v) \in B \times B$, then take an arbitrary $w \in C$; we have

$$
(u, w) \in B \times C \leq \alpha_{1}, \quad(w, v) \in C \times B \leq \beta_{1}
$$

and thus

$$
(u, v) \in \alpha_{1} \vee \beta_{1} .
$$

The case $(u, v) \in C \times C$ is similar. Therefore $\beta_{2} \leq \alpha_{1} \vee \beta_{1}$.

We have shown that $\alpha_{1} \vee \beta_{1}=\beta_{2}$. Next, since $\alpha_{1} \leq \alpha_{2} \leq \beta_{2}$, this implies that also $\alpha_{2} \vee \beta_{1}=\beta_{2}$.

Corollary 4.1. Quord $(A, f)$ contains a pentagon.

Let us summarize the obtained results.

Theorem 4.1. Let $(A, f)$ be a monounary algebra. The following conditions are equivalent:

(i) The lattice Quord $(A, f)$ is modular.

(ii) The lattice Quord $(A, f)$ is distributive.

(iii) Either $|A| \leq 2$ or $(A, f)$ is connected and there exists a cycle $C$ of $(A, f)$ such that $|A| \leq|C|+1$.

\section{REFERENCES}

[1] J. Berman, "On the congruence lattices of unary algebras," Proc. Amer. Math. Soc., vol. 36, pp. 34-38, 1972.

[2] I. Chajda and G. Czédli, "Four notes on quasiorder lattices," Math. Slovaca, vol. 46, no. 4, pp. 371-378, 1996.

[3] G. Czédli and A. Lenkehegyi, "On classes of ordered algebras and quasiorder distributivity," Acta Sci. Math. (Szeged), vol. 46, no. 1-4, pp. 41-54, 1983.

[4] M. Erné and J. Reinhold, "Intervals in lattices of quasiorders," Order, vol. 12, no. 4, pp. 375-403, 1995.

[5] G. Grätzer, General lattice theory, 2nd ed. Basel: Birkhäuser Verlag, 1998, new appendices by the author with B. A. Davey, R. Freese, B. Ganter, M. Greferath, P. Jipsen, H. A. Priestley, H. Rose, E. T. Schmidt, S. E. Schmidt, F. Wehrung and R. Wille.

[6] D. Jakubíková-Studenovská, "On completions of partial monounary algebras," Czechoslovak Math. J., vol. 38(113), no. 2, pp. 256-268, 1988.

[7] A. G. Pinus, "On lattices of quasi-orders on universal algebras," Algebra and Logic, vol. 34, no. 3, pp. 180-181, 1995.

[8] A. G. Pinus and I. Chajda, "Quasi-orders on universal algebras," Algebra and Logic, vol. 32, no. 3, pp. 164-173, 1993. 
[9] R. Pöschel and S. Radeleczki, "Endomorphisms of quasiorders and related lattices," in Contributions to general algebra. 18 . Klagenfurt: Heyn, 2008, pp. 113-128.

\section{Author's address}

D. Jakubiková-Studenovská

P. J. Šafárik University, Institute of Mathematics, Jesenná 5, 04154 Košice, Slovak Republic

E-mail address: danica.studenovska@upjs.sk 